\title{
Clinical factors and association with treatment modalities in patients with breast cancer and brain metastases who develop leptomeningeal disease
}

\author{
Akshjot Puri ( $\sim$ akshjot.puri11@gmail.com ) \\ Houston Methodist Hospital https://orcid.org/0000-0001-7187-7561
}

Charisma Mylavarapu

Houston Methodist Hospital

Jiaqiong $\mathrm{Xu}$

Houston Methodist Hospital

\section{Tejal A Patel}

LBJ Hospital: Lyndon B Johnson Hospital

\section{Bin S. Teh}

Houston Methodist Hospital

Ivo Tremont-Lukats

Houston Methodist

Jenny C. Chang

Houston Methodist Hospital

\section{Polly Niravath}

Houston Methodist Hospital

\section{Research Article}

Keywords: Breast cancer, brain metastases, leptomeningeal disease, risk factors, surgery, infratentorial

Posted Date: February 4th, 2022

DOI: https://doi.org/10.21203/rs.3.rs-1300783/v1

License: (c) (i) This work is licensed under a Creative Commons Attribution 4.0 International License. Read Full License 
Clinical factors and association with treatment modalities in patients with breast cancer and

brain metastases who develop leptomeningeal disease

Akshjot Puri, Charisma Mylavarapu, Jiaqiong Xu, Tejal A Patel, Bin S.Teh, Ivo Tremont-Lukats, Jenny C. Chang, Polly Niravath

Akshjot Puri, M.D. , Clinical Fellow, Hematology/Oncology

Houston Methodist Cancer Center

6445 Main St, Houston, TX 77030

Akshjot.puri11@gmail.com

Charisma Mylavarapu, D.O., Resident, Internal Medicine

Houston Methodist Hospital

6565 Fannin St, Houston, TX, 77030

Cmylavarapu@houstonmethodist.org

Tejal A. Patel, M.D., Breast Medical Oncology

The University of Texas MD Anderson Cancer Center

1515 Holcombe Blvd, Houston, TX, 77030

Tapatel1@mdanderson.org 
Bin S. Teh, M.D., Department of Radiation Oncology

Houston Methodist Hospital

6565 Fannin St, Houston, TX, 77030

bteh@houstonmethodist.org

Ivo W. Tremont, M.D., Department of Neuro Oncology

Houston Methodist Cancer Center

6445 Main St, Houston, TX 77030

Itremont@houstonmethodist.org

Jenny C. Chang, M.D., Breast Medical Oncology

Chair \& Director Cancer Center

Houston Methodist Cancer Center

6445 Main St, Houston, TX 77030

jcchang@houstonmethodist.org

Polly Niravath, M.D., Breast Medical Oncology Faculty

Houston Methodist Cancer Center

6445 Main St, Houston, TX 77030

paniravath@houstonmethodist.org 


\section{BIOSTATISTICIAN:}

Jiaqiong Xu, PhD, Center for Outcomes Research, Houston Methodist DeBakey Heart \& Vascular Center

Houston Methodist Research Institute

6445 Main St, Houston, TX 77030

sxu@houstonmethodist.org

Corresponding author: Akshjot Puri, MD, Clinical Fellow, Department of Hematology/Oncology,

Houston Methodist, 6445 Main St, Houston, TX 77030 USA

Phone: 3134375206

Fax: 7134418791

Akshjot.puri11@gmail.com

SPONSOR / FUNDING AGENCY

None

RUNNING HEAD

Risk for LMD in breast cancer and brain metastases

KEYWORDS:

Breast cancer, brain metastases, leptomeningeal disease, risk factors, surgery, infratentorial 


\section{ABSTRACT}

PURPOSE

Leptomeningeal disease (LMD) is an aggressive complication of metastatic breast cancer (MBC) with brain metastases (BM), with a short survival of weeks to months. Studies suggest that surgical resection of BM may increases the risk of LMD, especially in infratentorial metastases. In this retrospective study, we examine this and other factors which may be associated with increased risk of LMD.

\section{METHODS}

A database search at a single institution identified 178 patients with $\mathrm{MBC}$ and treated $\mathrm{BM}$ between 2007-2020. We collected demographic, clinical, radiographic and other treatment data. LMD was diagnosed by cerebrospinal fluid (CSF) cytology, neuroimaging, or both. Cox proportional hazards model was used.

\section{RESULTS}

After a median follow up of 8.5 months, 41 out of 178 patients (23\%) with BM developed LMD. Median time to develop LMD was 130 days. Mean age was 51.3 years. The number and size of the BM, hemorrhagic/cystic lesions, progressive/stable systemic disease, and extracranial metastases sites other than liver did not pose a higher risk of LMD. Infratentorial lesions $(H R=5.41)$ and liver metastases $(H R=2.28)$ had a higher risk of LMD. Patients who had any surgery did not have a higher risk for LMD (HR 1.13). The LMD group had a worse overall survival as compared to the non-LMD group.

\section{CONCLUSIONS}

Among $M B C$ patients with $B M$, infratentorial $B M$ and visceral liver lesions increase the risk of $L M D$ whereas local treatment modalities such as surgery and radiation do not. This data implies that local treatment strategy should not differ based on potential risk for LMD. 


\section{INTRODUCTION}

Improvements in the efficacy and outcomes of systemic therapies have led to significantly improved survival in patients with breast cancer. These advances have created a challenge with regards to management of brain metastases (BM) and leptomeningeal disease (LMD). There is an unmet need for therapies which can cross the blood brain barrier while minimizing neurotoxicity.

Adjuvant whole brain radiation therapy (WBRT) after stereotactic radiosurgery (SRS) or surgery for one to three BM in solid tumors reduced intracranial relapse and neurological deaths, but failed to improve the duration of functional independence and overall survival (OS) [1]. Additional evidence favors the use of SRS to the resection bed, achieving a high local control without the neurocognitive risks associated with WBRT [2]. Large BM (> 20mm) are associated with poor local control and more neurotoxicity with gamma knife (GK) [3] and owing to the mass effect, upfront surgical resection is often necessary. Fractionating a course of radiation can allow treatment of larger tumors that are often susceptible to CNS toxicity with better local control than single fraction radiosurgery [4]. Thus, the management of intracranial metastases entails an individualized multi-disciplinary approach.

LMD is a highly aggressive condition, defined as the infiltration of leptomeninges by metastatic carcinoma. It causes rapid neurological decline and a short survival of weeks to months [5]. Some authors have suggested that surgery may lead to leptomeningeal disease by intra-operative tumor spill [6]. In one retrospective study in patients with BM from breast cancer, surgery followed by SRS increased the 12 month rate of LMD compared to fractionated stereotactic radiation therapy (FSRT); (45\% vs $19 \%, p=0.048)$ [6]. In contrast, only 11 out of 126 (9\%) patients treated with SRS alone had LMD 12 months after diagnosis of BM [7]. Additional research shows preoperative SRS may reduce the risk of LMD in patients requiring surgical resection of BM compared with postoperative SRS (2 years: $16.6 \%$ vs 
$3.2 \%, P=.010$ ) with similar rates of OS and local/distant brain recurrence [8]. Also, patients with infratentorial BM have a higher risk for LMD $(p=0.012)[2]$.

In this cohort study, we included patients with breast cancer treated for BM. We then examined the factors that potentially predict LMD, such as surgery, location of brain metastases, and other clinical variables. We also analyzed survival and recurrence outcomes with various treatment modalities including surgery, SRS, or both.

Our primary objective was to determine if patients with BM who underwent surgical resection were at higher risk of LMD. Secondary objectives included: 1) to determine if infratentorial metastases pose a higher risk for developing $L M D, 2$ ) if other extracranial metastases posed an increased risk of $L M D, 3$ ) if the number and morphological features of BM (hemorrhagic/cystic lesions) had an association with LMD , 4) if there was an association between receptor status (HR and HER 2) and risk of LMD and 5) if there was an association of different treatment modalities with OS and risk of CNS recurrence.

\section{MATERIALS AND METHODS}

\section{Study design}

This study was retrospective, conducted at Houston Methodist Hospital, a tertiary care hos pital with a multi-disciplinary cancer center. The database was culled for patients with breast cancer (code C50) and treated BM (code C79.31). The study was approved by the Institutional review board at Houston Methodist. Patient confidentiality was maintained by de-identification, data storage in a password protected server, and access restricted only to the study investigators. The total cohort included 178 women seen in the outpatient or inpatient setting, older than 18 years of age with breast cancer and treated BM between September, 2007 to April, 2020. Only patients with sufficient demographical 
information, primary breast cancer pathology, neuroimaging at the time of diagnosis of BM, treatment details for BM and follow up were included. The exclusion criteria were patients with primary brain malignancy, BM from other primary cancers, prior WBRT, and history of intracranial surgery for another brain metastatic lesion. Clinical and pathological data extraction included patient demographics, performance status, clinical presentation, treatment course, and radiographic, dosimetric, recurrence, and survival data.

LMD was diagnosed by cerebrospinal fluid (CSF) cytology. If cytology was not available or was inconclusive, focal or diffuse leptomeningeal enhancement of the brain, spinal cord, cauda equina, cranial nerves (sensitivity 75\%) on the magnetic resonance imaging (MRI) was acceptable for diagnosis. For surgery of brain metastases, the extent of resection was classified as gross total or subtotal by postoperative MRI. Patients who underwent en bloc resection and piecemeal resection were included; however, patients with only stereotactic biopsy were excluded. Data on radiation modality, dose and frequency was collected and if radiation was administered alone or peri-operatively. Local recurrence of a brain metastases was defined as the development of new nodular contrast enhancement within $5 \mathrm{~mm}$ of the resection cavity in surgical patients or a $25 \%$ increase in tumor diameter on follow up MRI in patients treated with radiation [6]. Recurrence at another CNS site was defined as recurrence in the brain at any other site excluding local recurrence. Potential confounding factors in survival analysis were other comorbidities and deteriorating performance status with progression of disease. The patient follow-up closed in June 2020 for final data analysis.

\section{Statistical Analysis}

The number of cases meeting the inclusion criteria during the study period determined the sample size. Patients lost to follow up or with missing data were excluded. Baseline clinical, radiographic, and dosimetry data are summarized by LMD status. The summary statistics were means \pm SD for continuous 
variables, median and interquartile range (IQR) for skewed continuous variables, and absolute number and portions (percentages) for categorical variables. The Chi-square or Fisher's exact test (categorical variables) and the Student t-test or Mann-Whitney test (continuous variables) were used for hypothesis testing. The median time to develop LMD with different treatment modalities was compared using Kruskal-Wallis test. The incidence rate of LMD, OS, and incidence rate of local recurrence with $95 \%$ confidence intervals were calculated. Kaplan-Meier curves were constructed to estimate the OS and logrank test was used to compare the survivals between groups. A univariable Cox proportional hazards model was used to examine associations of clinical, radiographic, dosimetric variables, and surgery type with risk of developing LMD. Only variables with significance level of $p<0.05$ were incorporated in the multivariable analysis. Using backward model selection, we finalized the model when all variables were statistically significant. The same Cox proportional hazards model was used to examine the association of surgery type with OS and presence of local recurrence. The proportionality assumption of Cox models was verified by including time-dependent interaction of each covariate with event time in the model. All analyses were performed with STATA version 16 (StataCorp. 2019. Stata Statistical Software: Release 16. College Station, TX: StataCorp LLC). Statistical sig nificance was defined as two-tailed $p<0.05$ for all tests.

\section{RESULTS}

The search identified 374 patients using the breast cancer (code C50) and treated brain metastases (code C79.31). Out of these, 178 patients met the inclusion criteria for the study and analysis (Figure 1). The median age for the whole cohort was 49 years (mean $51.3 \pm 13.4$ years, IQR: 28 years-88 years) at the time of breast cancer diagnosis. One of the 178 patients was a male patient. In the whole cohort, 58.4\% were Caucasians, 19.1\% African-Americans, and 12.4\% Hispanics. The whole cohort had 20.3\% patients with $\mathrm{HR}+$ / HER2 - disease , $37.9 \%$ were HER2+ cancers and $41.8 \%$ had triple negative breast 
cancer (TNBC). There were $33.7 \%$ patients who had liver, $42.1 \%$ had lung, and $47.4 \%$ had secondary bone metastatic lesions. Table 1 lists additional demographic and descriptive details.

After a median follow up of 8.5 months since diagnosis of brain metastases (IQR: 54 days-21.2 months), 41 out of 178 patients (23\%) developed LMD. Two patients (4.8\%) were diagnosed with CSF cytology alone, 30 patients ( $73.2 \%)$ were diagnosed with neuroimaging alone and 9 patients $(22.0 \%)$ had evidence of LMD on both. The median age for those with LMD was 47 years (mean $47.9 \pm 12.3$ years, IQR: 38 years- 54 years) at the time of breast cancer diagnosis ( $p=0.057)$. All 41 patients with LMD were women. Systemic disease was progressive in $39.0 \%$, stable in $12.2 \%$ and $39.0 \%$ had no evidence of systemic disease at the time of diagnosis of BM. (Table 1)

The median number of brain metastatic lesions was 3 in both groups with the whole cohort having an IQR of 1-8, whereas in the LMD group the IQR was 1-9. The mean size of the largest brain lesion in the whole cohort was $2.45 \pm 1.45 \mathrm{~cm}$ and in the LMD group was $2.53 \pm 1.69 \mathrm{~cm}(\mathrm{p}=0.70)$. Twenty-two patients had hemorrhagic brain metastases but only 3 developed LMD (13.6 \%). Sixteen patients had cystic brain lesions and 3 developed LMD (18.8\%). In $29 \%$ of patients, the metastases were supratentorial, $24 \%$ were infratentorial, and $47.4 \%$ patients had supra and infratentorial metastases(Table 2 ).

For all patients, 44 (25.6\%) were treated with stereotactic radiation surgery (SRS) alone, 62 (36.0\%) received only whole brain radiation therapy (WBRT), 14 (8.1\%) underwent surgeryalone and 52 (30.2\%) had surgery in combination with perioperative radiotherapy (SRS or WBRT). In the LMD group, 5 (13.2\%) received SRS, 15 (39.5\%) had WBRT, 4 (10.5\%) had surgeryalone and $14(36.8 \%)$ had surgery in combination with perioperative radiation. Patients who had surgeryalone (HR 2.04; $p=0.29$ ) or in combination with perioperative radiotherapy (HR 2; $\mathrm{p}=0.19)$ did not have a higher risk for LMD. Of the 66 patients that underwent surgery, 58 (87.9\%) had en-block resection and eight (12.1\%) piecemeal resection. Two out of the 8 patients (25\%) who underwent piecemeal resection developed LMD; in our 
study piecemeal resection was not associated with increased risk of LMD (HR 0.89; $p=0.87)$. Table 3 summarizes the association of LMD with different treatment modalities. There was no statistically significant increased risk of LMD seen in those with infratentorial BM who underwent surgery as shown in table $4(p=0.230)$.

Out of 178 patients, 50 patients (28.1\%) developed local recurrences. Among these patients who locally recurred, 34\% had undergone previous SRS alone, 30\% had prior WBRT, $8 \%$ had undergone surgery alone and $28 \%$ had received surgery in combination with perioperative radiotherapy. There were 65 patients (36.5\%) who developed metastatic brain lesions at other sites in the CNS. Out of these $36.9 \%$ had received prior SRS, $29.2 \%$ had undergone WBRT previously, $6.1 \%$ had prior surgery alone and $27.7 \%$ underwent surgery in combination with perioperative radiotherapy. There was no statistically significant association between any of the treatment modalities with increased rate of local recurrence $(p=0.42)$, recurrence at other CNS sites $(p=0.16)$ or death $(p=0.67)$. Table 5 summarizes the outcomes of different treatment modalities; Figure 2 (suppl) shows survival association with different treatment modalities.

The median time (days) to develop LMD for the whole cohort was 130.5 (IQR: 0.01-504). For different treatment modalities they were 875 days (IQR: 504 days -1142 days) for SRS, 0.01 days (IQR: 0.01 days - 307 days) for WBRT, 1007 days (IQR: 225.5 days - 1744 days) for surgery alone and 97 days (IQR: 0.01 days -280 days) for those who underwent surgery with perioperative radiotherapy. There was no statistically significant association between any treatment modality and incidence of LMD $(p=0.085)$ (Table 6).

Univariable analysis $(\mathrm{HR}=1.91,95 \% \mathrm{Cl}: 1,3.64 ; \mathrm{p}=0.05)$ and multivariable analysis $(\mathrm{HR}=2.28,95 \% \mathrm{Cl}: 1.15$, 4.51; $p=0.018$ ) show the presence of liver metastases was significantly associated with development of LMD. Presence of both supra and infratentorial lesions simultaneously were associated with a higher 
risk for $L M D$ on univariable analysis $(H R=2.29,95 \% \mathrm{Cl}: 1.07,4.91 ; p=0.033)$. However, presence of only infratentorial lesions compared with supratentorial alone had a strong association with development of LMD by univariable $(\mathrm{HR}=4.55,95 \% \mathrm{Cl}: 1.85,11.18 ; \mathrm{p}=0.001)$ and multivariable $(\mathrm{HR}=5.41,95 \% \mathrm{Cl}: 2.18$, 13.45; $p<0.001)$ analysis. Age (HR 0.99; $P=0.29)$, African-American ethnicity (HR 1.32; $p=0.46),>3$ metastases (HR 1.21; $p=0.56$ ), size of the largest brain lesion (HR 1.06; $p=0.62$ ), hemorrhagic/cystic appearance (HR 0.99; $p=0.99)$, HR status (HR 0.82; $p=0.54) / H E R 2$ status of tumor (HR 0.58; $p=0.1$ ) or TNBC (HR 1.25; $p=0.49$ ) were not associated with development of LMD in this study. The breast cancer biomarkers were not associated with development of $\operatorname{LMD}(p=0.54, p=0.09$ and $p=0.48$ respectively), as seen in figures 4-6 (suppl). There were no significant associations for all other factors with LMD (Table 7). Overall, the LMD group had worse OS as compared to the non-LMD group ( $p=0.04)$ (Figure 3 suppl). Among 41 patients who developed LMD, there were 24 patients (58.54\%) who had LMD within 6 months and 31 (75.61\%) within 12 months of diagnosis of brain metastases.

\section{DISCUSSION}

The historical median OS in those with breast cancer and BM is 4 months, with a one year survival of $20 \%$ [9]. This has improved with recent advances in systemic treatments, with our data showing a median OS of 8.5 months and a one year survival of $39.9 \%$. With the advent of CNS-capable drugs like capecitabine, and novel drugs such as neratinib and tucatinib in patients with HER 2 positive BM, we anticipate the mean OS of these patients will continue to increase. Our study showed similar incidence of LMD as seen previously in literature where 41 out of 178 patients (23\%) developed LMD [10]. Our study corroborates that metastatic breast cancer patients with BM and LMD have a significantly worse survival that those with BM who do not have signs of LMD. Our study was performed to identify clinical risk factors which may help predict association with LMD. This will help in early diagnosis and initiating early treatment, thereby potentially increasing survival in this subset of metastatic breast cancer patients which are predicted to increase in number over the next few years. 
In our study the median age of patients who developed LMD was slightly younger ( 47.8 years) as compared to the patients who developed BM (51.3 years), but this was not statistically significant. Even though Caucasian women (104 out of $178,58.4 \%$ ) made up the majority of the whole cohort, only a smaller percentage developed LMD (24 out of 104 patients, 23.1\%). However, the incidence of LMD was higher in patients of African American origin; out of 34 patients with BM, 10 developed LMD (29.4\%). Due to the small numbers of the study population we are unable to conclude any statistically significant association between ethnicity and risk of LMD.

Previous studies have reported an increased incidence of LMD in patients with BM treated with surgery (HR 3.697, $p=0.02)[6]$. In contrast, this was not found to be significant in our study. Out of 178 patients with BM secondary to breast cancer, 66 patients (37.1\%) underwent surgery (with or without perioperative radiotherapy). Out of these 66 patients, 18 (27.3\%) developed LMD (HR 1.13 p=0.26). We also saw no statistically significant association with different treatment modalities including SRS, WBRT and surgery (with or without adjuvant radiotherapy) and rates of local recurrence, recurrence at other site in the CNS and death. The patients who underwent surgeryalone had 1-2 solitary brain lesions under $3 \mathrm{~cm}$ in size. However, other patients had a higher burden of CNS diseas e which explains the lower CNS recurrence risk seen with surgeryalone.

There is some evidence to support that there is a higher risk for LMD in patients with infratentorial brain metastatic lesions [2]. This was corroborated in our study, where we saw an increased risk for LMD in patient with infratentorial brain lesions (HR 5.41, $p<0.001$ ). Other tumor characteristics such as number of lesions, size of largest lesion and hemorrhagic/cystic character of BM did not translate into a higher risk of LMD.

There is no reported evidence in literature to support that increased burden of visceral metastasis has an increased incidence of LMD. In our study, we found that patients with liver metastases had a higher 
risk of developing LMD (HR 2.28, $p=0.018$ ). This was not found to be significant for those who had synchronous lung or skeletal metastatic lesions.

As per our data, none of the hormonal subtypes of breast cancer had an increased association with LMD. Our study population included an equally distributed proportion of patients $(40.3 \% \mathrm{HR}$ positive, 37.5\% HER2 positive and $41.8 \%$ TNBC patients) with BM. This may vary from previously reported prevalence numbers in literature due to the fact that some HER2 positive and TNBC patients may have a more aggressive disease course and may have not gotten treatment for their BM as they pursued comfort care status. This may have led to a selection bias in our patient population leading to a higher representation of $\mathrm{HR}$ positive patients, as patients with $\mathrm{BM}$ who did not receive any treatment (surgery/radiation) were not included in our study. As per a study out of 118 patients with LMD 35\% had lobular histology [11]. In our study, only 4 out of the 178 patients had lobular histology and 2 of these patients developed LMD. The small numbers of the patients do not allow us to make any inference regarding lobular histology and risk of LMD.

Interestingly, out of the 46 patients who had brain only metastatic disease with no evidence of systemic disease, 16 (34.8\%) developed LMD. This is in contrast to the 75 patients with systemically progressive disease at the time of development of BM where only 16 patients developed LMD (21.3\%). Out of these 75 patients with visceral progressive disease, 20 patients $(26.7 \%)$ had metastatic liver lesions, which as per our study poses a higher risk for LMD.

An important limitation of our study is the existing selection and medical surveillance bias. Only patients with available data regarding study variables and follow up information were included in the study. Patients who were lost to follow up or did not receive any treatment for their brain metastases or LMD and were transitioned directly to comfort care due to their disease being very aggressive or performance status being very poor were not included in our study. This could have an impact on the 
overall incidence and survival rates of patients with LMD. Also, LMD diagnosis was made by neuroimaging in 30 out of 41 patients (73.2\%) due to unavailability of CSF analysis. This may have lowered the sensitivity of LMD diagnosis as compared to the gold standard CSF study.

As per the results of our study, it is evident that among breast cancer patients with brain metastases those who develop LMD tend to be younger, with higher risk in Caucasians and African-American women; however, this was not statistically significant. The number, size, hemorrhagic/cystic character of brain lesions did not pose a higher risk of developing LMD. Our study data shows that patients with infratentorial metastatic brain lesions and those with visceral liver metastases have a statistically significant higher risk of LMD and could likely benefit by early diagnosis and treatment and perhaps have prolonged OS . Thus, it raises an important question regarding devising a planned imaging strategy for LMD screen in patients with breast cancer with BM and high risk features. The benefit of screening neuroimaging for LMD in metastatic breast cancer patient without neurological symptoms should be evaluated on a case by case basis. This study forms the ground work for future studies which can be performed at a multi-institutional platform, including larger number of patients and can provide corroborative evidence. The range of patients included in our study was broad, including one male patient with breast cancer and BM, ranging from 28 to 88 years with diverse ethnicities; representation of all breast cancer subtypes with most of the patients receiving standard of care treatments. The results of this study can be applied to other clinical settings of breast cancer patients as we included a cohort of patients which well represents the general metastatic breastcancer population in terms of age, sex, severity of disease and treatments. Any impacts of other factors like comorbidities, genetic differences in patients from other countries will need to be studied. 


\section{CONCLUSIONS}

With increasing novel treatments targeting brain metastases secondary to breast cancer we expect an increase in survival for a number of such patients. This is eventually likely to increase the incidence of complications of CNS involvement by breast cancer such as LMD. In our study there was no statistically significant difference in the rates of LMD, local recurrence, CNS recurrence at another site with surgery and/or radiation. We also identified clinical factors which may be associated with development of LMD. Patients with these risk factors such as infratentorial brain metastatic lesions and visceral liver metastases despite lack of neurological symptoms should be considered for screening neuroimaging to detect and treat early LMD. This study forms the ground work for future larger sized studies to analyze the role of dedicated screening and surveillance imaging for LMD in this subset of breast cancer patients with brain metastases who may be a at a higher risk to develop this aggressive infiltrative form of metastatic carcinoma

\section{CONFLICTS OF INTEREST}

None

SPONSOR / FUNDING AGENCY

None

\section{REFERENCES}

1. Kocher, M., et al., Adjuvant whole-brain radiotherapy versus observation after radiosurgery or surgical resection of one to three cerebral metastases: results of the EORTC 22952-26001 study. Journal of clinical oncology : official journal of the American Society of Clinical Oncology, 2011. 29(2): p. 134-141.

2. Ojerholm, E., et al., Stereotactic radiosurgery to the resection bed for intracranial metastases and risk of leptomeningeal carcinomatosis. J Neurosurg, 2014. 121 Suppl: p. 75-83. 
3. Shaw, E., et al., Single dose radiosurgical treatment of recurrent previously irradiated primary brain tumors and brain metastases: final report of RTOG protocol90-05. Int J Radiat Oncol Biol Phys, 2000. 47(2): p. 291-8.

4. Kim, Y.J., et al., Single-dose versus fractionated stereotactic radiotherapy for brain metastases. Int J Radiat Oncol Biol Phys, 2011. 81(2): p. 483-9.

5. Grossman, S.A., et al., Randomized prospective comparison of intraventricular methotrexate and thiotepa in patients with previously untreated neoplastic meningitis. Eastern Cooperative Oncology Group. J Clin Oncol, 1993. 11(3): p. 561-9.

6. Marcrom, S.R., et al., Focal Management of Large Brain Metastases and Risk of Leptomeningeal Disease. Advances in radiation oncology, 2019. 5(1): p. 34-42.

7. Trifiletti, D.M., et al., Leptomeningeal disease following stereotactic radiosurgery for brain metastases from breast cancer. J Neurooncol, 2015. 124(3): p. 421-7.

8. Patel, K.R., et al., Comparing Preoperative With Postoperative Stereotactic Radiosurgery for Resectable Brain Metastases: A Multi-institutional Analysis. Neurosurgery, 2016. 79(2): p. 27985.

9. Pestalozzi, B.C., et al., Identifying breast cancer patients at risk for Central Nervous System (CNS) metastases in trials of the International Breast Cancer Study Group (IBCSG). Ann Oncol, 2006. 17(6): p. 935-44.

10. Kim, H.J., et al., Clinical outcome of central nervous system metastases from breast cancer: differences in survival depending on systemic treatment. J Neurooncol, 2012. 106(2): p. 303-13.

11. Niwińska, A., H. Rudnicka, and M.J.M.o. Murawska, Breast cancer leptomeningealmetastasis: propensity of breast cancer subtypes for leptomeninges and the analysis of factors influencing survival. 2013. 30(1): p. 408. 


\section{STATEMENTS \& DECLARATION}

\section{FUNDING}

The authors declare that no funds, grants, or the other support were received during the preparation of this manuscript.

\section{COMPETING INTERESTS}

The authors have no relevant financial or non-financial interests to disclose.

\section{AUTHOR CONTRIBUTIONS}

All authors contributed to the study conception and design. Material preparation, data collection and analysis were performed by Akshjot Puri, Charisma Mylavarapu and Jiaqiong Xu. The first draft of the manuscript was written by Akshjot Puri and all authors commented on previous versions of the manuscript. All authors read and approved the final manuscript.

\section{DATA AVAILABILITY}

The datasets generated during and/or analysed during the current study are not publicly available so as to protect individual patient privacy, but are available from the corresponding author on reasonable request.

\section{ETHICS APPROVAL}

The study was approved by Institutional Review Board (IRB) at Houston Methodist Hospital. (IRB protocol \# 1210-0229)

\section{CONSENT TO PARTICIPATE}

Individual consent for this retrospective analysis was waived 
Figure 1: Consort diagram to show study enrollment

374 Patients assessed

for eligibility

\section{Excluded}

65 No brain metastases identified

65 No sufficient follow up information

41 Primary lung cancer

7 Primary brain malignancy

3 No breast cancer

3 Primary colorectal cancer

3 Primary ovarian cancer

2 Primary endometrial cancer

2 Primary renal cancer

1 Primary Urothelial cancer

1 Primary esophageal cancer

1 Primary prostate cancer

1 Primary vaginal squamous cell cancer

1 Cancer of unknown primary

178 Patients included

in the study and

analysis 
Figure 2: Survival association with different treatment modalities

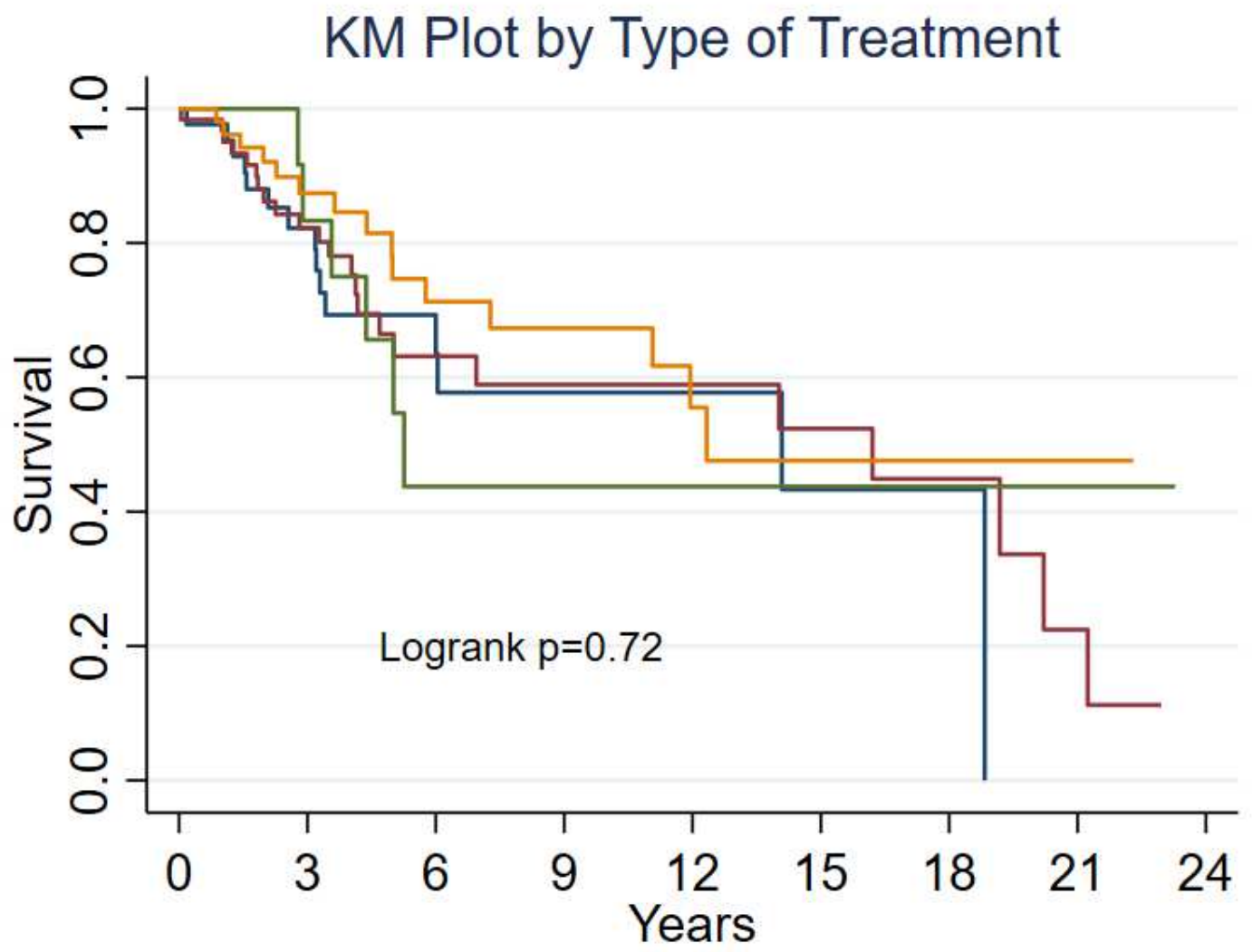

Number at risk

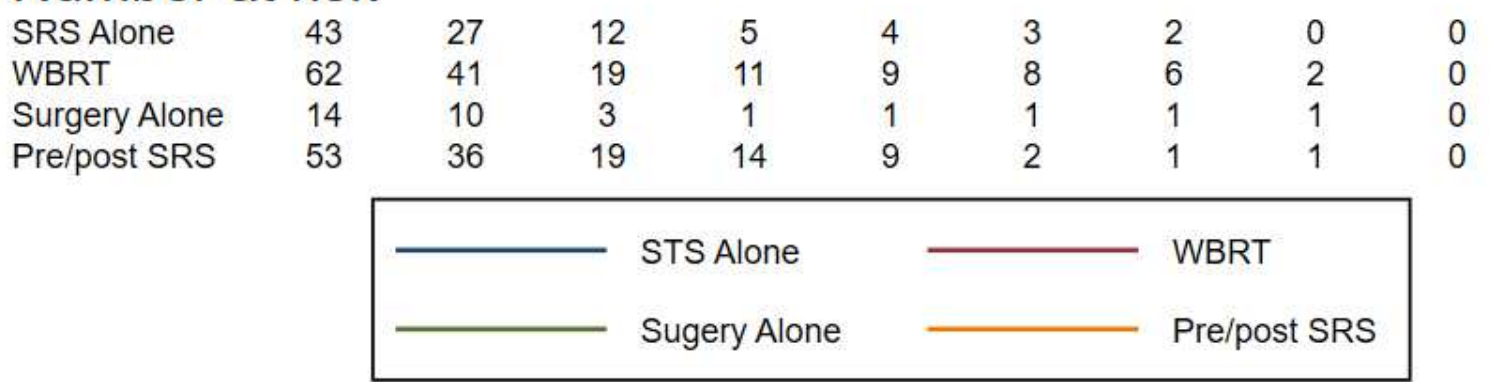


Figure 3: Survival association by presence of LMD

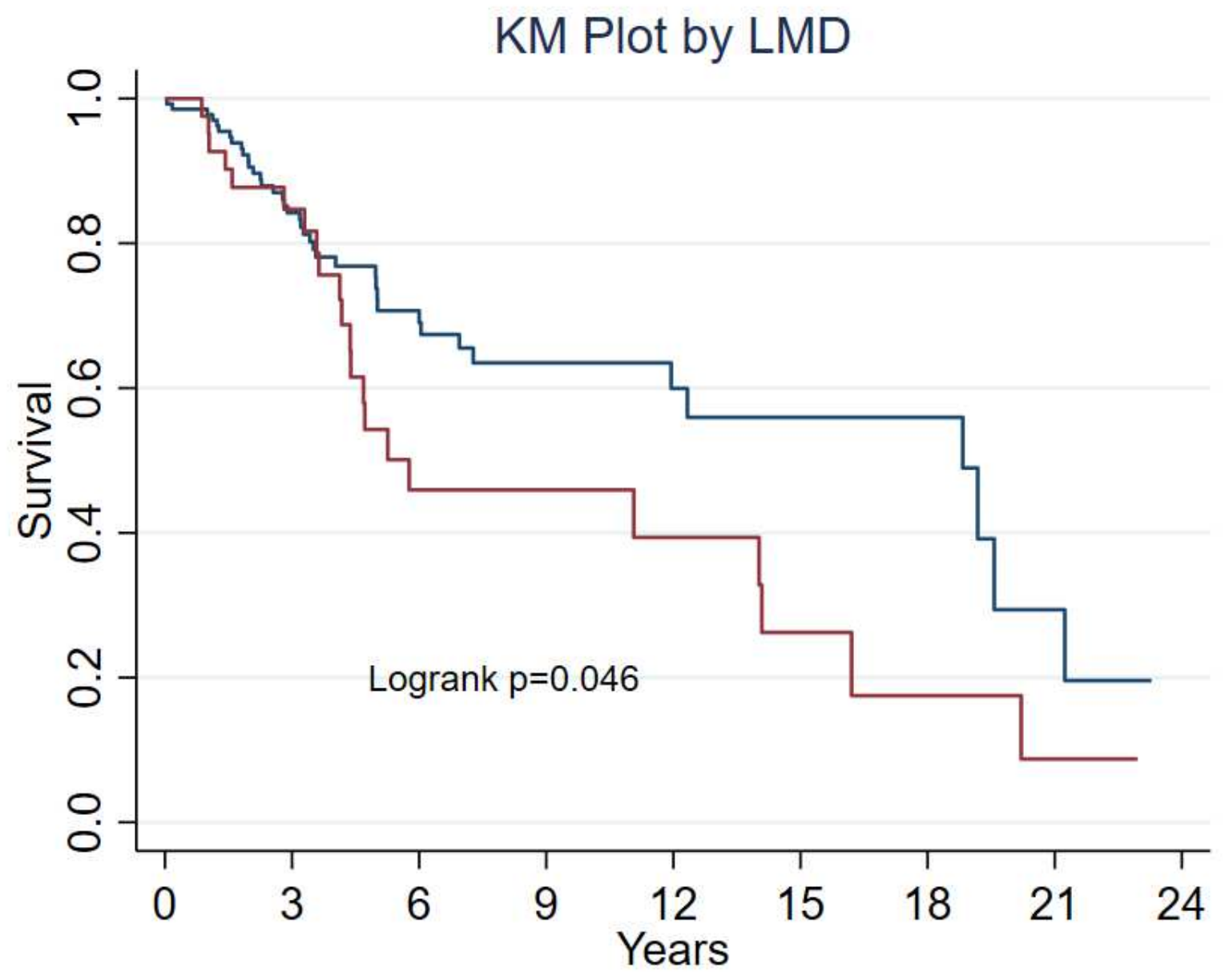

Number at risk

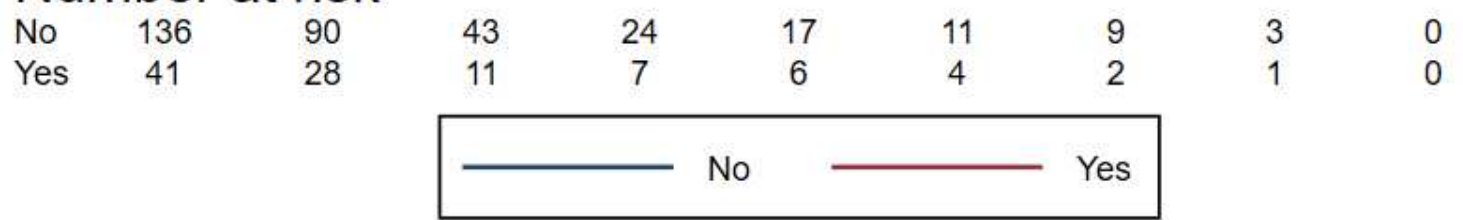


Figure 4: Development of LMD by hormone receptor status

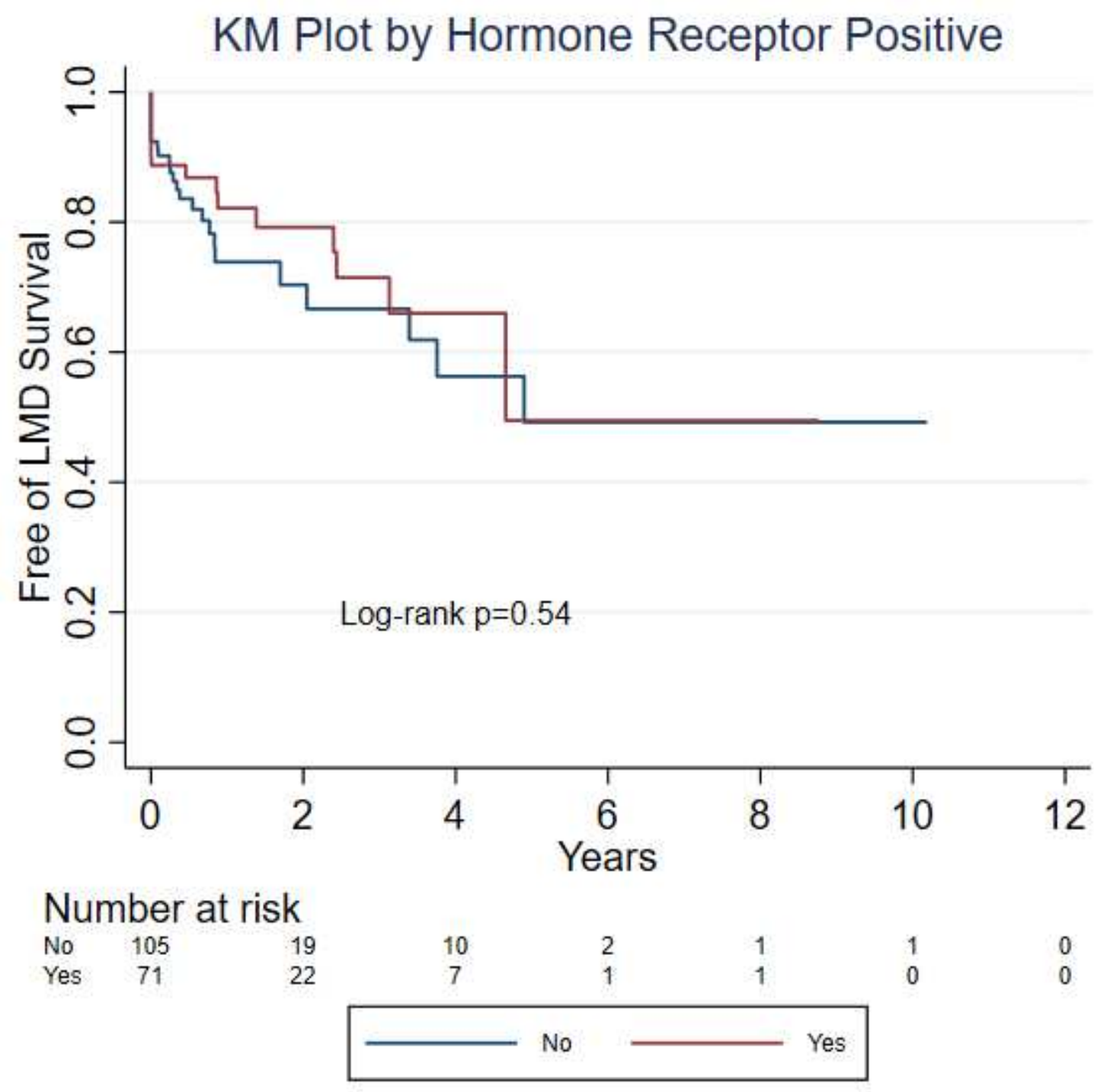


Figure 5: Development of LMD by HER2 status

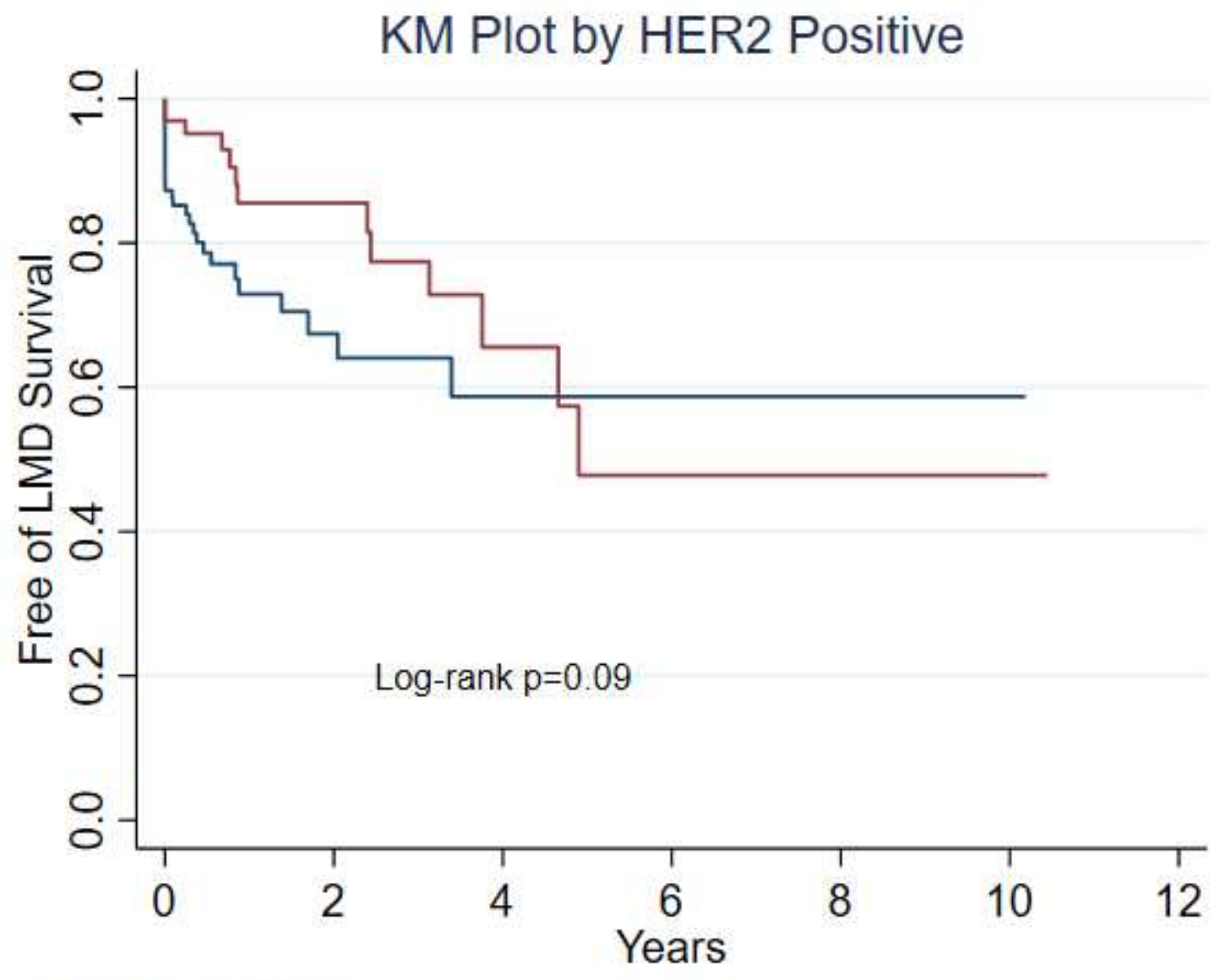

Number at risk

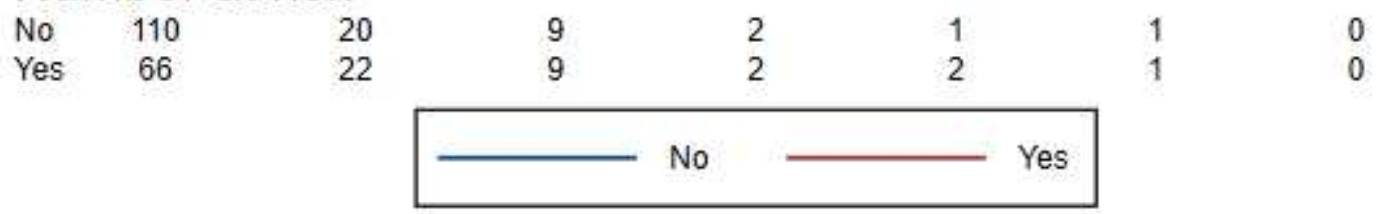


Figure 6: Development of LMD by triple negative status

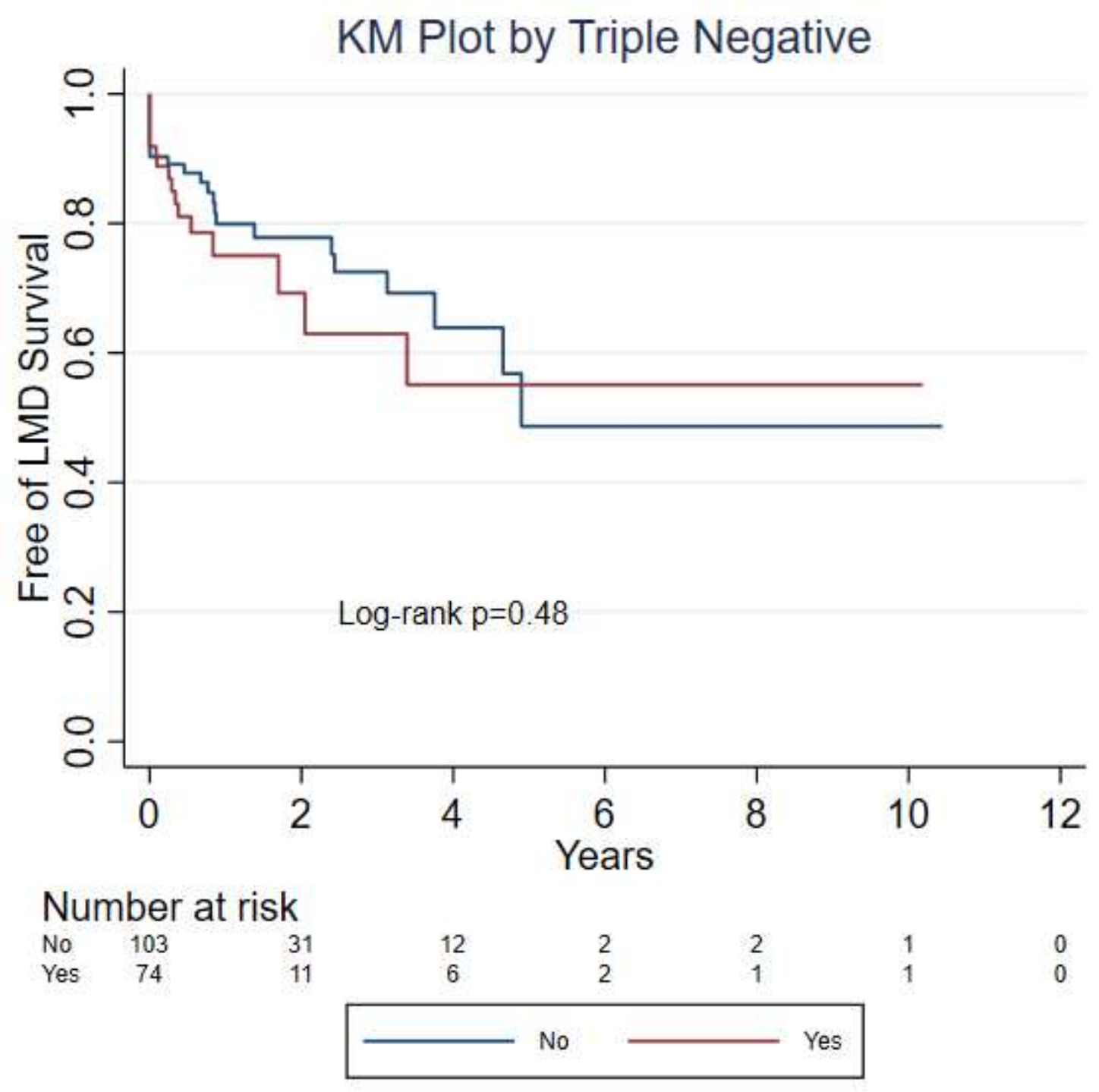


Table 1: Baseline characteristics of patients with brain metastases and those with LMD

\begin{tabular}{|c|c|c|c|c|}
\hline & \multirow{2}{*}{ Total } & \multicolumn{2}{|c|}{ LMD } & \multirow{3}{*}{ p-value } \\
\hline & & No & Yes & \\
\hline & $\mathrm{N}=178$ & $\mathrm{~N}=137$ & $\mathrm{~N}=41$ & \\
\hline Age & $51.34 \pm 13.37$ & $52.39 \pm 13.56$ & $47.85 \pm 12.25$ & 0.057 \\
\hline Female & 177 (99.44) & $136(99.27)$ & $41(100.00)$ & 1.00 \\
\hline Ethnicity & & & & 0.31 \\
\hline Caucasian & $104(58.43)$ & $80(58.39)$ & $24(58.54)$ & \\
\hline Hispanic & $22(12.36)$ & $20(14.60)$ & $2(4.88)$ & \\
\hline African American & $34(19.10)$ & $24(17.52)$ & $10(24.39)$ & \\
\hline Other & $18(10.11)$ & $13(9.49)$ & $5(12.20)$ & \\
\hline Liver Met & $59(33.71)$ & $43(32.09)$ & $16(39.02)$ & 0.45 \\
\hline Lung Met & $74(42.05)$ & $63(46.67)$ & $11(26.83)$ & 0.03 \\
\hline Bone Met & $83(47.43)$ & $66(49.25)$ & $17(41.46)$ & 0.48 \\
\hline HR Positive & $36(20.3)$ & $25(18.4)$ & $11(26.8)$ & 1.00 \\
\hline HER2 Positive & $67(37.9)$ & $54(39.7)$ & $13(31.7)$ & 0.46 \\
\hline Triple Negative & $74(41.8)$ & $57(41.9)$ & $17(41.5)$ & 1.00 \\
\hline Extra CNS disease & & & & 0.34 \\
\hline Stable & $34(19.32)$ & $29(21.48)$ & $5(12.20)$ & \\
\hline NED & 46 (26.14) & $30(22.22)$ & $16(39.02)$ & \\
\hline Progressive & $75(42.61)$ & $59(43.70)$ & $16(39.02)$ & \\
\hline Partial response & $8(4.55)$ & $7(5.19)$ & $1(2.44)$ & \\
\hline Mixed response & $7(3.98)$ & $5(3.70)$ & $2(4.88)$ & \\
\hline
\end{tabular}




\begin{tabular}{|l|l|l|l|l|}
\hline De Novo & $6(3.41)$ & $5(3.70)$ & $1(2.44)$ & \\
\hline
\end{tabular}

Data are presented as mean \pm SD or median (interquartile range) for continuous variables, and $\mathrm{n}(\%)$ for categorical variables. Chi-square or Fisher's exact test (categorical variables) and t-test or Mann-Whitney test (continuous variables) were used to compare patients who had LMD and not.

Table 2: Characteristics of the brain metastatic lesions and association with LMD

\begin{tabular}{|c|c|c|c|c|}
\hline \multirow{2}{*}{} & \multirow{2}{*}{ Total } & \multicolumn{2}{|c|}{ LMD } & \multirow{2}{*}{ p-value } \\
\cline { 3 - 4 } & & No & Yes & \\
\cline { 2 - 4 } & $\mathbf{N}=\mathbf{1 7 8}$ & $\mathbf{N = 1 3 7}$ & $\mathbf{N}=\mathbf{4 1}$ & \\
\hline Number of brain metastases & $3(1-8)$ & $3(1-8)$ & $2.5(1-9)$ & 0.57 \\
\hline By median cutoff point & & & & 1.0 \\
\hline$<=3$ & $100(58.14)$ & $78(58.21)$ & $22(57.89)$ & \\
\hline$>3$ & $72(41.86)$ & $56(41.79)$ & $16(42.11)$ & \\
\hline Largest metastatic lesion size (cm) & $2.45 \pm 1.45$ & $2.43 \pm 1.38$ & $2.53 \pm 1.69$ & 0.70 \\
\hline Hemorrhagic or Cystic & & & & 0.68 \\
\hline Hemorrhagic & $22(57.89)$ & $19(59.38)$ & $3(50.00)$ & \\
\hline Cystic & $16(42.11)$ & $13(40.63)$ & $3(50.00)$ & \\
\hline Location of Brain Met & & & & 0.025 \\
\hline Supratentorial & $75(43.10)$ & $64(47.06)$ & $11(28.95)$ & \\
\hline Infratentorial & $21(12.07)$ & $12(8.82)$ & $9(23.68)$ & \\
\hline Both & $78(44.83)$ & $60(44.12)$ & $18(47.37)$ & \\
\hline & & & & \\
\hline & & & & \\
\hline & & & & \\
\hline
\end{tabular}


Data are presented as mean \pm SD or median (interquartile range) for continuous variables, and $\mathrm{n}(\%)$ for categorical variables. Chi-square or Fisher's exact test (categorical variables) and t-test or Mann-Whitney test (continuous variables) were used to compare patients who had LMD and not.

Table 3: Association of LMD with different treatment modalities

\begin{tabular}{|c|l|l|l|l|}
\hline & \multicolumn{2}{|l|}{ LMD } & \\
\hline & Total & No & Yes & p-value \\
& $(\mathrm{N}=178)$ & $(\mathrm{N}=137)$ & $(\mathrm{N}=41)$ & \\
\hline Type of Treatment & & & & 0.21 \\
\hline SRS Alone & & & & \\
\hline WBRT & $44(25.6)$ & $39(29.10)$ & $5(13.2)$ & \\
\hline Surgery Alone & $62(36.0)$ & $47(35.07)$ & $15(39.5)$ & \\
\hline Any surgery & $14(8.1)$ & $10(7.46)$ & $4(10.5)$ & \\
\hline
\end{tabular}

Data are presented as $\mathrm{n}(\%)$. Fisher's exact test was used to compare patients who had LMD and not.

Table 4: LMD risk after surgical resection of supratentorial vs infratentorial brain lesions

\begin{tabular}{|l|l|l|l|l|}
\hline & N & LMD & No LMD & p-value \\
\hline Site of brain metastases & & & & 0.23 \\
\hline Supratentorial & 44 & $10(55.56 \%)$ & $34(73.91 \%)$ & \\
\hline Infratentorial & 20 & $8(44.44 \%)$ & $12(26.09 \%)$ & \\
\hline
\end{tabular}

Data are presented as $n(\%)$. Fisher's exact test was used to compare patients who had LMD and not. 
Table 5: Outcomes of different treatment modalities

\begin{tabular}{|l|c|c|c|c|}
\hline Type of Treatment & LMD & Local & CNS & Death \\
& $(n=38)$ & Recurrence & Recurrence \\
$(n=50)$ & $(n=65)$ & 15 \\
\hline SRS Alone & $5(13.2 \%)$ & $17(34 \%)$ & $24(36.9 \%)$ & $(25.4 \%)$ \\
\hline WBRT & 15 & $15(30 \%)$ & $19(29.2 \%)$ & 23 \\
\hline Surgery Alone & $(39.5 \%)$ & & $4(39.0 \%)$ \\
\hline Surgery with perioperative & $4(10.5 \%)$ & $4(8 \%)$ & $4(6.1 \%)$ & $6(10.2 \%)$ \\
\hline radiotherapy & 14 & $14(28 \%)$ & $18(27.7 \%)$ & 15 \\
\hline p-value & $(36.8 \%)$ & & 0.16 & 0.67 \\
\hline
\end{tabular}

Data are presented as $\mathrm{n}(\%)$. Fisher's exact test was used to compare patients who had events and not. 
Table 6: Time (days) to develop LMD with different treatment modalities

\begin{tabular}{|c|c|c|c|c|c|c|c|}
\hline $\begin{array}{l}\text { Type of } \\
\text { treatment }\end{array}$ & $\mathrm{N}$ & Mean & SD & Median & $\begin{array}{c}25^{\text {th }} \\
\text { percentile }\end{array}$ & $\begin{array}{c}75^{\text {th }} \\
\text { percentile }\end{array}$ & $p$-value \\
\hline & & & & & & & 0.085 \\
\hline SRS Alone & 44 & 466.98 & 532.59 & 875 & 90.5 & 709 & \\
\hline WBRT & 62 & 407.74 & 651.37 & 0.01 & 21 & 509 & \\
\hline Surgery Alone & 14 & 721.29 & 951.66 & 1007 & 54 & 1192 & \\
\hline $\begin{array}{l}\text { Surgery with } \\
\text { perioperative } \\
\text { radiotherapy }\end{array}$ & 52 & 648.52 & 778.54 & 97 & 143 & 981.5 & \\
\hline
\end{tabular}

Kruskal-Wallis test was used to compare medians among the type of treatment. 
Table 7: Hazards ratio $(95 \% \mathrm{Cl})$ for developing LMD from Cox proportional hazards regression model

\begin{tabular}{|c|c|c|c|c|}
\hline & \multicolumn{2}{|l|}{ Univariable } & \multicolumn{2}{|l|}{ Multivariable } \\
\hline & $\mathrm{HR}(95 \% \mathrm{Cl})$ & $p$-value & $\mathrm{HR}(95 \% \mathrm{Cl})$ & $\begin{array}{l}\mathrm{p}- \\
\text { value }\end{array}$ \\
\hline Age & $0.99(.96,1.01)$ & 0.29 & & \\
\hline \multicolumn{5}{|l|}{ Race } \\
\hline White & Ref & & & \\
\hline Hispanic & $0.38(0.09,1.62)$ & 0.19 & & \\
\hline African American & $1.32(0.63,2.77)$ & 0.46 & & \\
\hline Other & $1.09(0.42,2.86)$ & 0.86 & & \\
\hline Number of brain metastases $>3$ & $1.21(.63,2.32)$ & 0.56 & & \\
\hline Tumor size $(\mathrm{cm})$ & $1.06(.85,1.32)$ & 0.62 & & \\
\hline Cystic vs hemorrhagic & $0.99(.19,5.02)$ & 0.99 & & \\
\hline \multicolumn{5}{|l|}{ Location of Brain Met } \\
\hline Supratentorial & Ref & & Ref & \\
\hline Infratentorial & $4.55(1.85,11.18)$ & 0.001 & $5.41(2.18,13.45)$ & $<0.001$ \\
\hline Both & $2.29(1.07,4.91)$ & 0.033 & $2.05(0.95,4.43)$ & 0.069 \\
\hline \multicolumn{5}{|l|}{ Location of Large Brain Met } \\
\hline Supratentorial & Ref & & & \\
\hline Infratentorial & $2.20(1.12,4.34)$ & 0.023 & & \\
\hline Equivocal & $0.91(0.21,3.90)$ & 0.9 & & \\
\hline Liver Met & $1.91(1,3.64)$ & 0.05 & $2.28(1.15,4.51)$ & 0.018 \\
\hline Lung Met & $0.65(.32,1.31)$ & 0.23 & & \\
\hline
\end{tabular}




\begin{tabular}{|l|l|l|l|l|}
\hline Bone Met & $0.97(.52,1.82)$ & 0.93 & & \\
\hline HR Positive & $0.82(.44,1.55)$ & 0.54 & & \\
\hline HER2 Positive & $0.58(.3,1.12)$ & 0.1 & & \\
\hline Triple Negative & $1.25(.67,2.34)$ & 0.49 & & \\
\hline Type of Treatment & & & & \\
\hline SRS Alone & Ref & & & \\
\hline WBRT & $2.40(0.87,6.60)$ & 0.091 & & \\
\hline Surgery Alone & $2.04(0.54,7.64)$ & 0.29 & & \\
\hline Pre/post SRS & $2.00(0.72,5.56)$ & 0.19 & & \\
\hline Any surgery & $1.13(.59,2.15)$ & 0.71 & & \\
\hline Piece meal vs. Block & $0.89(.2,3.86)$ & 0.87 & & \\
\hline
\end{tabular}

Multivariable model included those significant $(p<0.05)$ from univariable model. 